\title{
Subject Index to Volume 8
}

\section{Abstracts}

Program Abstracts of the XVI Congress of Neurological Sciences, 185 (May)

Adrenoleucodystrophy

191 (May) Abs

Amyotrophic Lateral Sclerosis

Abnormal Proximal Axons of Clarke's Neurons in Sporadic Motor Neuron Disease, 173 (May)

A cluster of amyotrophic lateral sclerosis, 199 (May) Abs

Role of circulating immune complexes in multiple sclerosis and amyotrophic lateral sclerosis, 189 (May) Abs

\section{Aneurysms}

Effect of Therapy on Cerebral Blood Flow Following Aneurysm Surgery, 27 (Feb)

\section{Anoxia}

Anoxic Tonic Seizures Due to Asthma; A Serious Complication in Adults, 177 (May)

Anticoagulant

Spinal Subdural Hematoma in Association with Anticoagulant Therapy, 87 (Feb)

\section{Anticonvulsant}

Effects of ethyl ester derivatives of valproic acid metabolites on pentylenetetrazol seizures in mice, 259 (Aug)

The effect of single anticonvulsants on fasting plasma ammonia and amino acids in children, 201 (May) Abs

Valproic Acid and Plasma Levels of Primdone and Derived Phenobarbital, 91 (Feb)

Valproic Acid; steady-rate pharmacokinetics in 43 pediatric patients with seizures, 193 (May) Abs

Aplysia

What the marine mollusc Aplysia can tell the neurologist about behavioral neurophysiology, 275 (Nov)

\section{Apraxia}

Respiratory inhibitory apraxia; Clinical and computerized tomographic study, 194 (May) Abs

Arachnoid Cysts

Suprasellar arachnoid cysts: Investigation and management, 192 (May) Abs

Arteriovenous Malformations

Cerebral arteriovenous malformations in childhood, 187 (May) Abs

Global Arteriovenous Malformation of the Cervical Region, 41 (Feb)

The Computerized Tomographic Appearance of Angiographically Occult Arteriovenous Malformations of the Brain, 7 (Feb)

\section{Astrocytoma}

Extraneural Metastases of a Cerebral Astrocytoma, 115 (May)

\section{Barbiturates}

Further observations on the role of barbiturate protection in global cerebral ischemia in the mongolian gerbil, 195 (May) Abs
Blood Brain Barrier

The blood brain barrier and folate deficiency, 194 (May) Abs

\section{Book Reviews}

93 (Feb), 203 (May), 265 (Aug), 339 (Nov)

Brain Abscess

Meningitis and brain abscess due to clostridium perfringens and clostridium paraputrificum following orbital trauma, 309 (Nov)

\section{Bromocriptine}

Method of Addition of Bromocriptine to the Drug Regime of Patients with Advance Parkinson's Disease, 31 (Feb)

\section{Carbon Monoxide}

Delayed Encephalopathy Following Carbon Monoxide Intoxication, 77 (Feb)

\section{Carotid Artery Stenosis}

External carotid artery stenosis after extracranial-intracranial anastomoses, 187 (May) Abs

Intracranial internal carotid artery stenosis, 195 (May) Abs

Carotid Cavernous Fistula

Treatment of carotid cavernous fistula, 187 (May) Abs

\section{Carotid Endarterectomy}

Intraoperative regional cerebral blood flow during carotid endarterectomy, 235 (Aug)

Neuropsychological Changes Following Carotid Endarterectomy, 127 (May)

\section{Carotid Stenosis}

Enhanced detection of carotid stenosis by doppler using spectral analysis, 198 (May) Abs

Prophylactic carotid artery repair on the asymptomatic side contralateral to previously operated symptomatic carotid stenosis, 189 (May) Abs

Seizures as a manifestation of TIA's in bilateral carotid stenosis, 188 (May) Abs

The natural history of the asymptomatic carotid bifurcation lesion: Implications for management, 189 (May) Abs

Cerebellar Infarction

Ventricular drainage for cerebellar infarction, 192 (May) Abs

\section{Cerebral Blood Flow}

Cerebral blood flow assessment of patients with extracranial-intracerebral shunts, 197 (May) Abs

Cerebral blood flow in bilateral carotid occlusion, 198 (May) Abs

Cerebral blood flow in profound intraoperative hypotension: Correlation with preand post-operative measurements, 193 (May) Abs

Effect of Therapy on Cerebral Blood Flow following Aneurysm Surgery, 27 (Feb)

Intraoperative regional cerebral blood flow during carotid endarterectomy, 235 (Aug)

\section{Cerebral Infarcts}

The Computed Tomographic Assessment of Brain Infarcts, 121 (May)

\section{Cerebral Ischemia}

Further observations on the role of barbiturate protection in global cerebral ischemia in the mongolian gerbil, 195 (May) Abs

\section{Cerebrovascular Disease}

$\mathrm{C} 2$ deficiency and stroke, 187 (May) Abs

Topographic electroencepalographic study of occlusive cerebrovascular disease following EC/IC bypass operation, 197 (May) Abs

\section{Cerebrovascular Permeability}

Cerebrovascular permeability in mechanically induced hypertension, 215 (Aug)

Clonazepam

Myoclonies du voile associées à une diminution de l'acide 5-hydroxy-indole-acétique céphalo-rachidien et répondant au clonazepam, 51 (Feb)

\section{Computed Tomography}

Pitfalls in Posterior Fossa CT Scanning: An Illustrative Case Report, 155 (May)

The Computed Tomographic Assessment of Brain Infarcts, 121 (May)

The Computerized Tomographic Appearance of Angiographically Occult Arteriovenous Malformations of the Brain, 7 (Feb)

\section{Cordectomy}

Cordectomy - Clinical-pathological correlation in seven cases, 188 (May) Abs

\section{Cortical Localization}

Paul Flechsig's System of Myelogenetic Cortical Localization in the Light of Recent Research in Neuroanatomy and Neurophysiology - Part I, I (Feb)

$$
\text { - Part II, } 95 \text { (May) }
$$

\section{Creutzfeldt - Jakob Disease}

Creutzfeldt-jakob disease presenting as conversion hysteria, 198 (May) Abs

\section{Dr. Derek Denny-Brown}

An Appreciation, 271 (Nov)

\section{Dopamine}

Dopamine Uptake Capacity of Platelets from People at Risk for Huntington's Chorea, 167 (May)

\section{Dystonia}

Conference report - The Torsion Dystonias: A summary of the Third Dystonia Work shop, 255 (Aug)

Post-hemiplegic dystonia: A clinical and computerized tomographic correlative study, 191 (May) Abs

Characteristic long-latency reflex abnormalities in dystonia and rigidity, 200 (May) Abs

\section{Electromyography}

Quantitative EMG of the APB, 200 (May) Abs

The Crossed Adductor Reflex in Humans: An EMG Study, 81 (Feb)

\section{Electroretinogram}

Abnormal electroretinograms and visual evoked potentials in chronic papilledema using time-difference analysis, 243 (Aug)

An electroretinal and visual evoked potential study in Friedreich's Ataxia, 289 (Nov) 


\section{Endodermal Sinus Tumors}

Intracranial endodermal sinus tumours, 193 (May) Abs

Epilepsy

Anoxic Tonic Seizures due to Asthma: A Serious Complication in Adults, 177 (May) Differences in sexual and genital ictal patterns suggest sexual dimorphism in temporal lobe epilepsy, 193 (May) Abs

Effects of ethyl ester derivatives of valproic acid metabolites on pentylenetetrazol seizures in mice, 259 (Aug)

Is ictal fear a feature of generalized seizures? 201 (May) Abs

Low blood and cerebrospinal fluid thiamine values in phenytoin treated epileptics, 201 (May) Abs

Seizures as a manifestation of TIA's in bilateral carotid stenosis, 188 (May) Abs

Valproic Acid and Plasma Levels of Primidone and Derived Phenobarbital, 91 (Feb)

\section{Evoked Potential}

Abnormal electroretinograms and visual evoked potentials in chronic papilledema using time-difference analysis, 243 (Aug)

An electroretinal and visual evoked potential study in Friedreich's Ataxia, 289 (Nov)

Evoked Potentials in Multiple Sclerosis, 15 (Feb)

Influence of muscle strength on somatosensory evoked potentials in the cat, 199 (May) Abs

Normalization of the somatosensory evoked potential in incomplete spinal cord injuries, 199 (May) Abs

Serial pattern visual evoked response studies in multiple sclerosis, 190 (May) Abs

Somatosensory evoked responses in patients with unilateral cerebral hemisphere lesions, 200 (May) Abs

Use of trigeminal nerve stimulation to evoke SEP's in Multiple Sclerosis suspects, 190 (May) Abs

Eye Movements

Task Dependent Variations of Ocular Lateropulsion in Wallenberg's Syndrome, 21 (Feb)

\section{Facial Nerve}

Facial nerve paralysis, 201 (May) Abs

Folate Deficiency

The blood brain barrier and folate deficiency, 194 (May) Abs

\section{Friedreich's Ataxia}

An electroretinal and visual evoked potential study in Friedreich's Ataxia, 289 (Nov) Friedreich's Ataxia with Nephrotic Syndrome and Convulsive Disorder: Clinical and Neurophysiological Studies with Renal and Nerve Biopsies and an Autopsy, 55 (Feb)

\section{Glioblastoma Multiforme}

Glioblastoma multiforme with pulmonary, osseous and spinal epidural implantations, 197 (May) Abs

\section{Glioma}

A simple and reproducible experimental in Vivo Glioma model, 325 (Nov)

Brain Stem glioma in childhood; Acute hemiplegic onset, 263 (Aug)
Guillain-Barre Syndrome

Treatment of Fisher's Variant of GuillainBarre Syndrome by Exchange Transfusion, 49 (Feb)

\section{Head Injury}

Changes in gray matter density from cranial computed tomography in severe head injury, 198 (May) Abs

University of Toronto head injury study, 196 (May) Abs

\section{Hemifacial Spasm}

Hemifacial spasm: Cause and Cure, 188 (May) Abs

Hemorrhage

Conservative or surgical management of intra-cerebral spontaneous hemorrhage - A retrospective clinical appraisal, 187 (May) Abs

Herpes Simplex

Isolation of Herpes Simplex virus from the central nervous system (CNS) of latently infected mice following immunosuppression, 198 (May) Abs

\section{Huntington's Chorea}

A Case of Huntington's Chorea with Unilateral Ectopic Gray Matter, 181 (May)

Dopamine Uptake Capacity of Platelets from People at Risk for Huntington's Chorea, 167 (May)

\section{Hydrocephalus}

Continuous intracranial pressure recording and intraventricular infusion studies in normal pressure hydrocephalus, 192 (May) Abs

\section{Hypertension}

Cerebrovascular permeability in mechanically induced hypertension, 215 (Aug)

Intracerebral arteriolar permeability to lanthanum, 194 (May) Abs

\section{Kindling}

Effects of cinromide, a new anticonvulsant, on cortex - and amygdala - kindled seizures in the rat, 193 (May) Abs

The effect of low frequency and direct current stimulation on the Kindling Phenomenon in rats, 249 (Aug)

The effect of several intertrial intervals on the $1 \mathrm{~Hz}$ interference effect, $61(\mathrm{Feb})$

Levodona

Chronic Levodona and Renal Function, 139 (May)

\section{Magladery John William}

86 (Feb)

Medulloblastoma

Adult medulloblastoma, 196 (May) Abs

\section{Meningitis}

Meningitis and brain abscess due to clostridium perfringens and clostridium paraputrificum following orbital trauma, 309 (Nov)

\section{Mental Retardation}

Recent advances in mental retardation - the fragile X chromosome, 191 (May) Abs

Metastatic Tumours

Multiple cerebral lesions and metastases in CT scan; experience of 106 cases, 197 (May) Abs
Migraine

Further study of dietary migraine, 191 (May) Abs

Migraine and partial seizures with complex symptomatology in children: An association?, 188 (May) Abs

Minimal Brain Dysfunction

Motor side effects with pemoline in minimal brain dysfunction syndrome, 191 (May) Abs

\section{Movement}

Motor learning in different kinds of movement, 200 (May) Abs

\section{Multiple Sclerosis}

Abnormal adaptation of visual contrast sensitivity in Multiple Sclerosis patients, 227 (Aug)

Conference Report - International symposium on Multiple Sclerosis, 333 (Nov)

Evoked Potentials in Multiple Sclerosis, 15 (Feb)

HLA typing in MS sibling pairs, 190 (May) Abs

Immune regulation in multiple sclerosis: T cell surface marker analysis, 187 (May) Abs

Immunofluorescent Staining of Rat Brain Glial Cells with Multiple Sclerosis Serum, 143 (May)

Multifocal CT enhancement in MS, 190 (May) Abs

Multiple Sclerosis and Associated Dieseases: A Relationship to Diabetes Mellitus, 35 (Feb)

Neurenteric Cysts of the Spinal Cord Mimicking Multiple Sclerosis, 159 (May)

Occult fifth nerve dysfunction in Multiple Sclerosis, 221 (Aug)

Role of circulating immune complexes (CIC) in multiple sclerosis and amyotrophic lateral sclerosis, 189 (May) Abs

Serial pattern visual evoked response studies in multiple sclerosis, 190 (May) Abs

Spinal Cord Swelling in Multiple Sclerosis, 151 (May)

The predictive value of CSF electrophoresis in "possible" multiple sclerosis, 189 (May) Abs Use of trigeminal nerve stimulation to evoke SEP's in Multiple sclerosis suspects, 190 (May) Abs

\section{Muscular Dystrophy}

Fukuyama's Disease, 199 (May) Abs

Prevalence and incidence of muscular dystrophy in Alberta, Canada, 191 (May) Abs

Myasthenia Gravis

Factors associated with relapse of myasthenia gravis after plasma exchange therapy, 198 (May) Abs

The Effect of Plasma-pheresis on PostThymectomy Ocular Dysfunction, 169 (May)

\section{Myoclonus}

Myoclonies du voile associées à une diminutuion de lacide 5-hydroxy-indole-acétique céphalo-rachidien et répondant au clonazepam, 51 (Feb)

Myopathy

Congenital structural myopathies, 199 (May) Abs

Neonatal myotubular myopathy: Neuropathy and failure of postnatal maturation of fetal muscle, 313 (Nov) 


\section{Myotonic Dystrophy}

Impaired insulin effect on the fibroblast surface membrane in myotonic dystrophy, 190 (May) Abs

\section{Narcolepsy}

Life effects of narcolepsy in 180 patients from North America, Asia, and Europe compared to matched controls, 299 (Nov)

\section{Nerve Biopsy}

Friedreich's Ataxia with Nephrotic Syndrome and Convulsive Disorder: Clinical and Neurophysiological Studies with Renal and Nerve Biopsies and an Autopsy, 55 (Feb)

\section{Nerve Conduction}

Temperature effects on human compound action potentials: computer simulation to define mechanisms, 201 (May) Abs

\section{Nerve Grafts}

Peripheral nerve grafts to the optic nerve in rats, 199 (May) Abs

Neurenteric Cysts

Neurenteric Cysts of the Spinal Cord Mimicking Multiple Sclerosis, 159 (May)

\section{Neuropathy}

Porphyric neuropathy: An ultrastructural and quantitative case study, 281 (Nov)

Clinical progression of Giant-Axonal Neuropathy over a twelve year period, 321 (Nov)

Plasma exchange in chronic demyelinating neuropathy, 189 (May) Abs

Chronic neuropathy associated with $\operatorname{lgM}$ antibody reactive to peripheral nerve myelin, 190 (May) Abs

Experimental Porphyric Neuropathy: A Preliminary Report, 105 (May)

The etiology of sciatic neuropathies, 199 (May) Abs

Sciatic nerve palsy due to endometriosis, 202 (May) Abs

\section{Neurophysiology}

What the marine mollusc Aplysia can tell the neurologist about behavioral neurophysiology, 275 (Nov)

\section{Neuroradiology}

Tracking down the oblique orbital (innominate) line, 198 (May) Abs

\section{Nystagmus}

Positional Vertigo and Nystagmus of Central Origin, 133 (May)

\section{Optic Nerve}

Multiple Regression Annalysis of Diagnostic Predictors in Optic Nerve Disease, 67 (Feb)

Pain

Peripheral nerve stimulation for relief of chronic pain, 192 (May) Abs

\section{Papilledema}

Abnormal electroretinograms and visual evoked potentials in chronic papilledema using time-difference analysis, 243 (Aug)

\section{Paraplegia}

The effect of prostacyclin in experimental traumatic paraplegia in rats, 188 (May) Abs

Parkinson's Disease

Chronic Levodona and Renal Function, 139 (May)

Method of Addition of Bromocriptine to the Drug Regime of Patients with Advanced Parkinson's Disease, 31 (Feb)

\section{Pelizaeus-Merzbacher Disease}

$\mathrm{X}$-Linked hereditary ataxic diplegia and Pelizaeus-Merzbacher Disease: The borderland revisited, 191 (May) Abs

\section{Pituitary Tumour}

Apoplexy, hemorrhage and necrosis in pituitary adenomata, 189 (May) Abs

Pituitary basophilism 1981: Tumour and "Pseudotumour" in Cushing's Disease, 189 (May) Abs

\section{Plasmapheresis}

Factors associated with relapse of myasthenis gravis after plasma exchange therapy, 198 (May) Abs

Plasma exchange in chronic demyelinating neuropathy, 189 (May) Abs

The Effect of Plasmapherseis on PostThymectomy Ocular Dysfunction, 169 (May) Treatment of Fisher's Variant of GuillainBarre Syndrome by Exchange Transfusion, 49 (Feb)

\section{Polymyositis}

Polymyositis Presenting with Distal and Assymmetrical Weakness, 147 (May)

\section{Porphyria}

Experimental Porphyric Neuropathy: A Preliminary Report, 105 (May)

Porphyric neuropathy: An ultrastructural and quantitative case study, 281 (Nov)

Porphyrin Neurotoxicity

Porphyrin neurotoxicity in vitro, 194 (May) Abs

\section{Radiotherapy}

An assessment of superfractionation radiotherapy in a murine model system and a prospective clinical trial, 195 (May) Abs

Reflexes

The Crossed Adductor Reflex in Humans: An EMG Study, 81 (Feb)

\section{Regeneration}

Axons from neurons in the cerebral hemispheres of adult rats grow in PNS grafts, 195 (May) Abs

Experimental elongation of mammalian spinal and medullary neurons, 195 (May) Abs

\section{Schizophrenia}

Structural Lesions of the Brain in Young Schizophrenics, 73 (Feb)

\section{Seizures}

Migraine and partial seizures with complex symptomatology in children: An association?, 188 (May) Abs

Shunting

Experience with lumboperitoneal shunting, 192 (May) Abs

Sleep Apnea

Lateral decubitus and subsidence of sleepinduced apneas (SIA): Therapeutic implications, 194 (May) Abs

Sleep apnea and impaired peripheral chemosensitivity in an adult patient with hindbrain malformation, 194 (May) Abs

\section{Spinal Cord Injury}

Atlanto-axial trauma with spinal cord injury, 196 (May) Abs
Halo devices in the treatment of acute cervical spinal cord injury: the Sunnybrook experience, 200 (May) Abs

Normalization of the somatosensory evoked potential in incomplete spinal cord injuries, 199 (May) Abs

Oxygenated fluorocarbon perfusion as treatment of acute spinal cord compression injury, 195 (May) Abs

Spondylosis in acute cervical spinal cord and spinal column injuries, 196 (May) Abs

Spondylosis in the etiology and outcome of cervical spinal cord injuries, 200 (May) Abs

\section{Spinal Cysts}

Post surgical extradural spinal cysts, 196 (May) Abs

Spinal Metastasis

Intradural extramedullary spinal metastasis, 196 (May) Abs

\section{Spinal Stenosis}

Lumbar Stenosis; Analyis of factors affecting outcome in 81 surgical cases, 295 (Nov)

\section{Subarachnoid Hemorrhage}

Chronic vasospasm in monkeys after large subarachnoid hemorrhage, 202 (May) Abs

CT scan and serial cerebral blood flow (CBF) measurement in the management of cerebral ischemic complications following subarachnoid hemorrhage, 197 (May) Abs

Subdural Empyema

Pasteurella Multocida Subdural Empyema: A Case Report, 163 (May)

\section{Subdural Hematoma}

Morbidity, mortality and recurrence rates of subdural hematomas in CT era, 197 (May) Abs

Spinal Subdural Hematoma in Association with Anticoagulant Therapy, 87 (Feb)

Syphillis

Luetic middle cerebral artery stenosis, 199 (May) Abs

Syringomy elia

Management of syringomyelia - a pathophysiological approach, 192 (May) Abs

\section{Thymectomy}

The Effect of Plasmapheresis on PostThymectomy Ocular Dysfunction, 169 (May)

\section{Torticollis}

Effect of L-tryptophan on spasmodic torticollis, 305 (Nov)

Selective peripheral denervation for spasmodic torticollis, 188 (May) Abs

\section{Transient Ischemic Attacks}

Topographic electroencephalographic study of TIAs in asymptomatic period, 187 (May) Abs

Trigeminal Ganglion

Human gasserian ganglion in tissue culture, 190 (May) Abs

\section{Trigeminal Neuralgia}

Comparison of microvascular decompression and percutaneous rhizotomy in the surgical treatment of trigeminal neuralgia - report of additional cases and further follow-up, 188 (May) Abs

Trigeminal Neuralgia: A comparison of the results of percutaneous rhizotomy and microvascular decompression, 207 (Aug) 


\section{Valproic Acid}

Efficacy and toxicity of valproic acid in patients with complex partial seizures, 201 (May) Abs

Valproic Acid: First-dose and steady-state pharmacokinetics in children with seizures, 201 (May) Abs

Valproic Acid producing a reye-like syndrome, 201 (May) Abs

Vertigo

Positional Vertigo and Nystagmus of Central Origin, 133 (May)
Vestibular Dysfunction

Ewald's second law as a basis for the localization of unilateral labyrinthine loss in humans, 193 (May) Abs

Vestibular Function

Can the vestibulo-ocular reflex be isolated for quantitative diagnosis? 202 (May) Abs

\section{Visual Contrast Sensitivity}

Abnormal adaptation of visual contrast sensitivity in Multiple Sclerosis patients, 227 (Aug)

\section{Wallenberg's Syndrome}

Task Dependent Variations of Ocular Lateropulsion in Wallenberg's Syndrome, 21 (Feb)

\section{Writer's Cramp}

Idiopathic dysgraphia (writer's cramp), 198 (May) Abs 